\title{
Evaluation of oxidant stress and antioxidant defense in discrete brain regions of rats exposed to $900 \mathrm{MHz}$ radiation
}

\author{
Narayanan $\mathrm{SN}^{1}$, Kumar $\mathrm{RS}{ }^{1}$, Kedage $\mathrm{V}^{2}$, Nalini $\mathrm{K}^{3}$, Nayak $\mathrm{S}^{4}$, Bhat $\mathrm{PG}^{5}$ \\ Department of Physiology, Melaka Manipal Medical College (Manipal Campus),Manipal University, Manipal, India. \\ sareeshnn@yahoo.co.in
}

\begin{abstract}
Aim: In the current study, the effects of $900 \mathrm{MHz}$ radio-frequency electromagnetic radiation (RF-EMR) on levels of thiobarbituric acid-reactive substances (TBARS), total antioxidants (TA), and glutathione S-transferase (GST) activity in discrete brain regions were studied in adolescent rats.

Materials and methods: Thirty-six male Wistar rats (6-8 weeks old) were allotted into three groups $(n=12$ in each group). Control group (1) remained undisturbed in their home cage; sham group (2) was exposed to mobile phone in switch off mode for four weeks; RF-EMR-exposed group (3) was exposed to $900 \mathrm{MHz}$ of RF-EMR (1 $\mathrm{hr} /$ day with peak power density of $146.60 \mu \mathrm{W} / \mathrm{cm}^{2}$ ) from an activated Global System for Mobile communication (GSM) mobile phone (kept in silent mode; no ring tone and no vibration) for four weeks. On 29th day, behavioral analysis was done. Followed by this, six animals from each group were sacrificed and biochemical parameters were studied in amygdala, hippocampus, frontal cortex, and cerebellum.

Results: Altered behavioral performances were found in RF-EMR-exposed rats. Additionally, elevated TBARS level was found with all brain regions studied. RF-EMR exposure significantly decreased TA in the amygdala and cerebellum but its level was not significantly changed in other brain regions. GST activity was significantly decreased in the hippocampus but, its activity was unaltered in other brain regions studied.

Conclusion: RF-EMR exposure for a month induced oxidative stress in rat brain, but its magnitude was different in different regions studied. RF-EMR-induced oxidative stress could be one of the underlying causes for the behavioral deficits seen in rats after RF-EMR exposure (Fig. 5, Ref. 37). Text in PDF www.elis.sk.

Key words: radio-frequency electromagnetic radiations, mobile phone, reactive oxygen species, adolescent rat.
\end{abstract}

Mobile phone use has boomed out in recent years with an estimated 4.6 billion subscriptions globally (1). It has become an integral part of modern telecommunications. In many countries, over $50 \%$ of the population uses mobile phones, and in some parts of the world, mobile phones are the most reliable or the only phones available (1). These gadgets use radiofrequency waves for transmitting data. The frequency band used for this varies from country to country. Generally the GSM (Global System for Mobile Communications) mobile phones use 900/1800 MHz frequency bands. Unlike ionizing radiation such as X-rays or gamma rays, radiofrequency fields can neither break chemical bonds nor cause ionization in the human body (1). Although this fact is true and

${ }^{1}$ Department of Physiology, Melaka Manipal Medical College (Manipal Campus), Manipal University, Manipal, India, ${ }^{2}$ Department of Molecular and Cellular Biochemistry, Indiana University, Bloomington, Indiana, USA, ${ }^{3}$ Department of Biochemistry, Kasturba Medical College, Manipal University, Manipal, India, ${ }^{4}$ Department of Anatomy, Melaka Manipal Medical College (Manipal Campus), Manipal University, Manipal, India, and ${ }^{5}$ Division of Biotechnology, School of Life Sciences, Manipal University, Manipal, India

Address for correspondence: S.N. Narayanan, Department of Physiology, Melaka Manipal Medical College (Manipal Campus), Manipal University, Manipal 576 104, India.

Phone: +91.9844897190 , Fax: +91.820 .2571905$

Acknowledgements: The authors are sincerely grateful to Indian Council of Medical Research (ICMR), New Delhi, for providing financial assistance (No.5/10/FR/21/2011-RHN) to carry out this research project. well known to everyone, the interaction between this radiation and biological system is a major concern. The possible health effects of mobile phone RF-EMR are bothering the whole world because of the uncontrollable growth of the industry over the globe. Very little is known about the effects of these radiations on biological systems. Adding more complexity to this situation, the International Agency for Research on Cancer (IARC) of World Health Organization (WHO) has classified RF-EMR emitted from mobile phones to be carcinogenic to humans (2). Growing body of research suggests the adverse effect of RF-EMR on various organs such as skin (3), testis (4) and thyroid gland (5). It also alters learning and memory $(6,7,8)$, hippocampal distribution of calbindin and glial fibrillary acidic protein (GFAP), immunoreactivity (9), emotionality (10) and brain glucose metabolism (11). Although there are number of reports on the effects of RF-EMR on various systems (especially on behavioral alteration in rats), no satisfactory mechanism has been proposed to explain the biological effects of these radiations on various systems. Reactive oxygen species (ROS) might play a role. ROS have been involved in a wide variety of cellular functions but they can be both essential and highly toxic to cellular homeostasis (12). ROS exert their cytotoxic effect by peroxidation of membrane phospholipids leading to change in permeability and loss of membrane integrity (13). Several reports suggest that RFEMR could alter the free radical metabolism in tissue in vivo and induce oxidative stress, but controversies stay on in many of these reports and several questions remain unanswered $(14,15,16,17)$. 
In the present scenario, it is important to elucidate whether the behavioral and other effects seen in rats after RF-EMR exposure are due to oxidative stress. In other words, the crucial questions are as follows: Does RF-EMR exposure induce oxidative stress in rat brain? If so, how does it affect various brain regions? Does it affect different brain regions equally or differently? Which area of the brain is affected the most due to oxidative stress induced by RF-EMR? Can this be correlated with the behavioral changes seen in rats after RF-EMR exposure? The current study is a preliminary attempt to answer these questions and to suggest an underlying cause for behavioral effects seen in rats due to RF-EMR exposure. We therefore investigated the effects of $900 \mathrm{MHz}$ RF-EMR on the levels of thiobarbituric acid-reactive substances (TBARS), total antioxidants (TA), and glutathione S-transferase (GST) activity in discrete brain regions (amygdala, hippocampus, frontal cortex, and cerebellum) of adolescent rats.

\section{Materials and methods}

\section{Animals}

All procedures used in the study were approved by the Institutional Animal Ethics Committee (IAEC). Albino rats of Wistar strain (6-8 weeks old, 150-160 g of weight) were used for the experiment. The rats were bred in the Central Animal Research Facility (CARF) and maintained on a 12-hr light/dark cycle (light on: 07:00-19:00) in a well ventilated temperature-controlled $\left(23^{\circ} \mathrm{C}\right.$ $\pm 2{ }^{\circ} \mathrm{C}$ ) room. They were housed in polypropylene cages $(3$ per cage) measuring $41 \mathrm{~cm} \times 28 \mathrm{~cm} \times 14 \mathrm{~cm}$ and fed with water and food ad libitum. In order to reduce the litter effects on dependent measures, rats in each experimental group were sampled from 8 different litters.

\section{Study design}

The study was conducted on 36 male Wistar rats. Animals were randomly allotted into three groups of 12 rats in each group. Animals from control group remained in their home cage for four weeks. Those from sham group were exposed to a mobile phone (in switch-off mode; $1 \mathrm{hr}$ /day for four weeks) placed in a wood bottom bamboo wire mesh cage in the center of the home cage. Animals from RF-EMR group were exposed to RF-EMR (900 $\mathrm{MHz}$ ) from an active GSM (Global System for Mobile communications) mobile phone (kept in silent mode; no ring tone and no vibration) for $1 \mathrm{hr} /$ day for four weeks. The placement of the mobile phone during exposure was same as mentioned above. After the experimental period (on $29^{\text {th }}$ day), behavioral analysis was done. Followed by this, six animals from each group were sacrificed to study biochemical indexes (such as TBARS, TA, and GST activity) in amygdala, hippocampus, frontal cortex, and cerebellum.

\section{$900 \mathrm{MHz}$ RF-EMR exposure and power dosimetry}

All groups of rats were kept under the same environmental conditions during the experiments. The method of mobile phone RF-EMR exposure and dosimetry is the same as described before $(8,18)$. Briefly, RF-EMR exposed group was exposed to $900 \mathrm{MHz}$ radiation from an active mobile phone. RF-EMR exposure by the downlink frequency band ( 890 to $915 \mathrm{MHz}$ with basic band of 900 $\mathrm{MHz}$ ) of GSM was studied. The exposure of rats was achieved by placing the phone in a wood bottom bamboo wire mesh cage (12 $\mathrm{cm} \times 7 \mathrm{~cm} \times 7 \mathrm{~cm}$ ) in the center of the home cage (which has 3 rats in it). This was done carefully to prevent the rats from getting in direct contact with the device. A level 4 GSM mobile phone with a permitted power level of $2 \mathrm{~W}$ (with SAR specification $1.58 \mathrm{~W} /$ $\mathrm{kg}$ ) was used for this purpose. Animals were exposed to $900 \mathrm{MHz}$ RF-EMR by giving 50 missed calls or unattended calls (within one hour) per day for 28 days. The phone was kept in silent mode (no ring tone and no vibration) and was allowed to ring $\sim 45$ seconds during each call, followed by a 15 -second interval before next call. This process was repeated 50 times. A spectrum analyzer (SPECTRAN HF-2025E with MCS Real-Time Spectrum Analyzer Software, Aaronia AG, Germany) was used to determine the power in the vicinity of mobile phone. The peak power density recorded from the vicinity of the mobile phone (3 $\mathrm{cm}$ away from the phone) was found to be $146.60 \mu \mathrm{W} / \mathrm{cm}^{2}$. When the cell phone was not activated but in 'ON' mode, the peak power density was found to be $2.109 \mathrm{nW} / \mathrm{cm}^{2}$ (18).

\section{Sample preparation and biochemical determination of oxidative stress}

Animals were sacrificed by cervical dislocation under light ether anesthesia. Brains were immediately removed and washed in ice-cold physiological saline repeatedly. Brain tissues of interest (amygdala, hippocampus, frontal cortex, and cerebellum) were carefully dissected out over an ice-cold watch glass (19). Each of the brain regions (right and left regions, in case of amygdala, hippocampus, and frontal cortex) were pooled, blotted, and weighed. These were then placed in chilled $0.01 \mathrm{~mol} / \mathrm{L}$ phosphate buffer $(\mathrm{pH}$ 7.4) for homogenizing. Tissue homogenate was prepared using 10 $\mathrm{ml}$ of PBS for every gram of tissue. This was done using a tissue homogenizer (ROTEK, Ambala, INDIA). The homogenate was then centrifuged at $3000 \mathrm{rpm}$ for $15 \mathrm{~min}$. The supernatant was collected and transferred to an eppendorf tube and again centrifuged at $12000 \mathrm{rpm}$ for $30 \mathrm{~min}$. The supernatant thus obtained was used to determine various biochemical indexes using the Genesys 10 UV spectrophotometer. To avoid experimenter bias, an independent person coded the samples before subjecting them to various biochemical determinations. Protein concentrations of the tissue homogenates were determined by the standard method of Bradford et al (20) using bovine serum albumin as standard, and the values were expressed in $\mathrm{mg} / \mathrm{ml}$. Satoh's method (21) was followed to determine the TBARS levels, and the method described by Koracevic et al (22) was used to determine total antioxidants. For determining Glutathione S-transferase activity in various brain regions, method described by Beutler was followed (23).

\section{Statistical analysis}

Values of various groups are expressed as Mean \pm S.E.M. One way analysis of variance test (ANOVA) with Tukey's post-hoc test was used to determine statistical difference between groups. $p$ value $<0.05$ was considered statistically significant. 
260-266

\section{Results}

Altered behavioral performances were found in RF-EMR exposed rats (published elsewhere; (18)). In the present report, altered biochemical indexes seen in discrete brain regions of rats exposed to RF-EMR are presented.

\section{Biochemical indexes in the amygdala}

TBARS levels were significantly elevated in the amygdala of rats exposed to RF-EMR. In control/sham animals, it was $\sim 20$ $\mathrm{pmol} / \mathrm{mg}$ of tissue protein, but in the RF-EMR exposed rats, it was elevated more than two times. The approximate mean value reached $50 \mathrm{pmol} / \mathrm{mg}$ of tissue protein (Fig. 1A: Control/Sham vs RF-EMR; ** $\mathrm{p}<0.01, \partial \partial \partial \mathrm{p}<0.001$; ANOVA, Tukey's post hoc tests). TA was significantly decreased in the RF-EMR group compared to control/sham groups (Fig. 1B: Control vs RF-EMR; $* \mathrm{p}<0.05$, Sham vs RF-EMR; $\partial \mathrm{p}<0.05$ ANOVA, Tukey's post hoc tests). GST activity was not significantly different in RF-EMR group in comparison to control/sham groups (Fig. 1C).
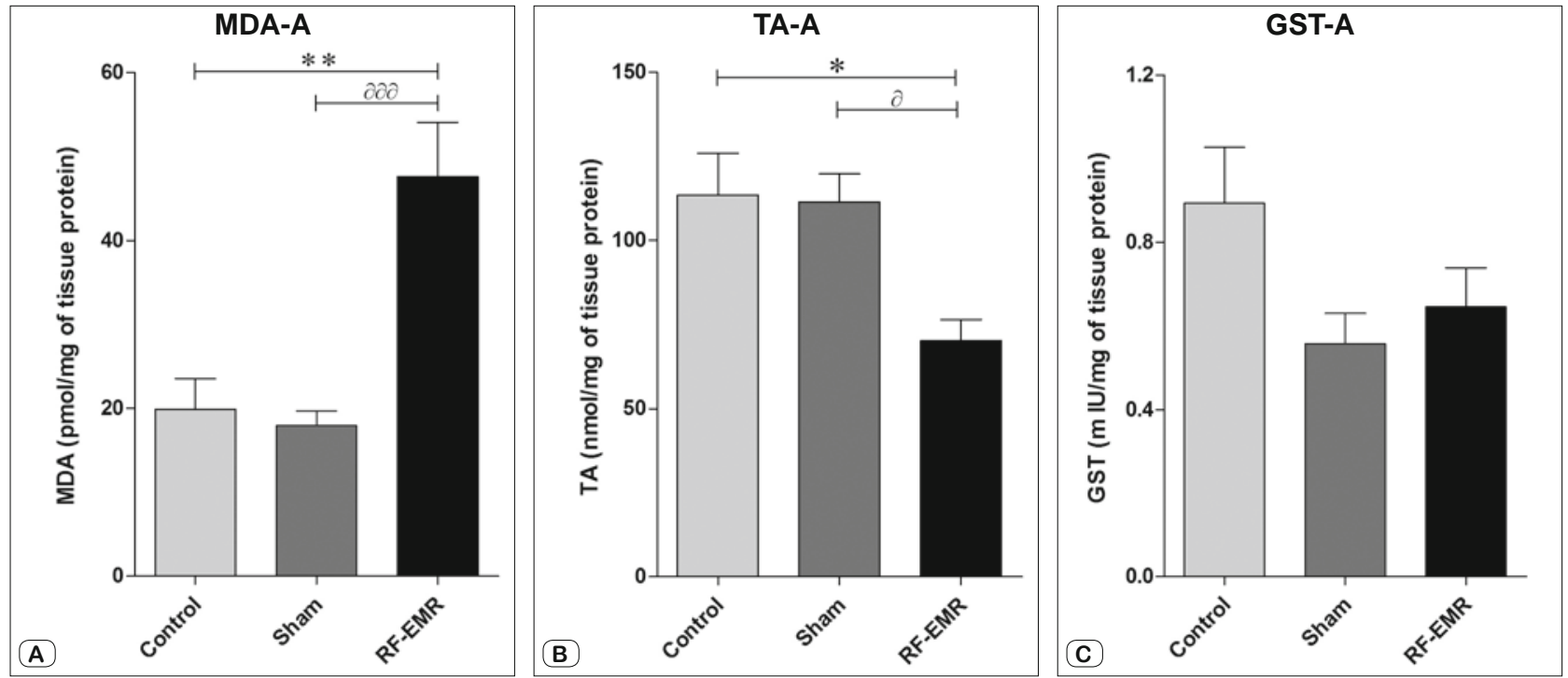

Fig. 1. Levels of (A) thiobarbituric acid reactive substances, (B) total antioxidants, and (C) Glutathione S-transferase activity in the amygdala. TBARS levels were significantly elevated and TA levels were significantly decreased in the amygdala. GST activity was not changed significantly in RF-EMR group in comparison to control/sham groups. (Note: MDA-A - Malondialdehyde levels in amygdala, TA-A - Total antioxidants in amygdala, and GST-A - Glutathione S-transferase activity in amygdala respectively). $* * p<0.01, \partial \mathrm{p}<0.05, \partial \partial \partial \mathrm{p}<0.001$.
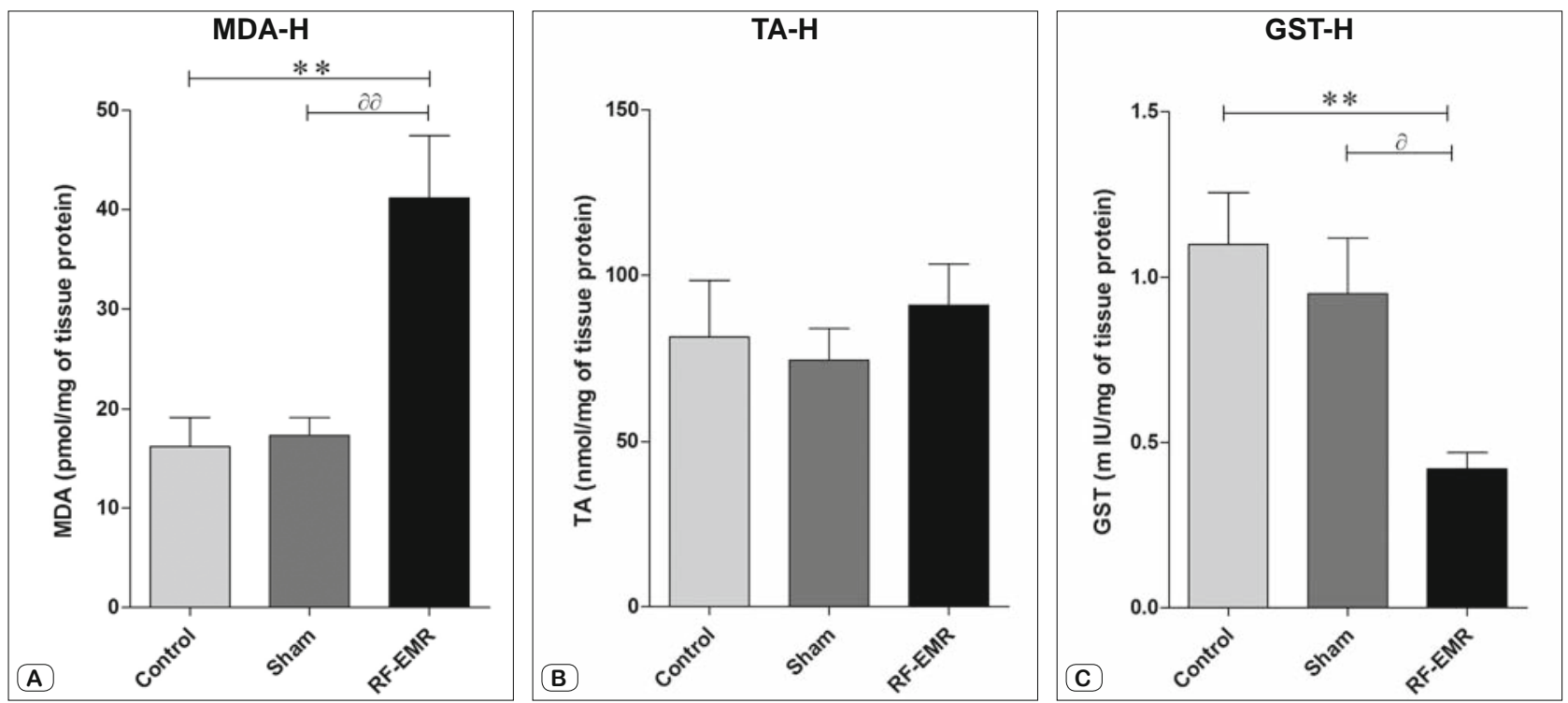

Fig. 2. Levels of thiobarbituric acid reactive substances (A), total antioxidants (B), and Glutathione S-transferase activity (C) in the hippocampus. TBARS levels were elevated $\sim 2$ times in RF-EMR exposed group than control/sham groups. TA remained unaltered and GST activity was significantly decreased in comparison to control/sham groups. (Note: MDA-H - Malondialdehyde levels in hippocampus, TA-H - Total antioxidants in hippocampus, and GST-H - Glutathione S-transferase activity in hippocampus respectively). $* * p<0.01, \partial \partial p<0.01, \partial p<0.05$. 

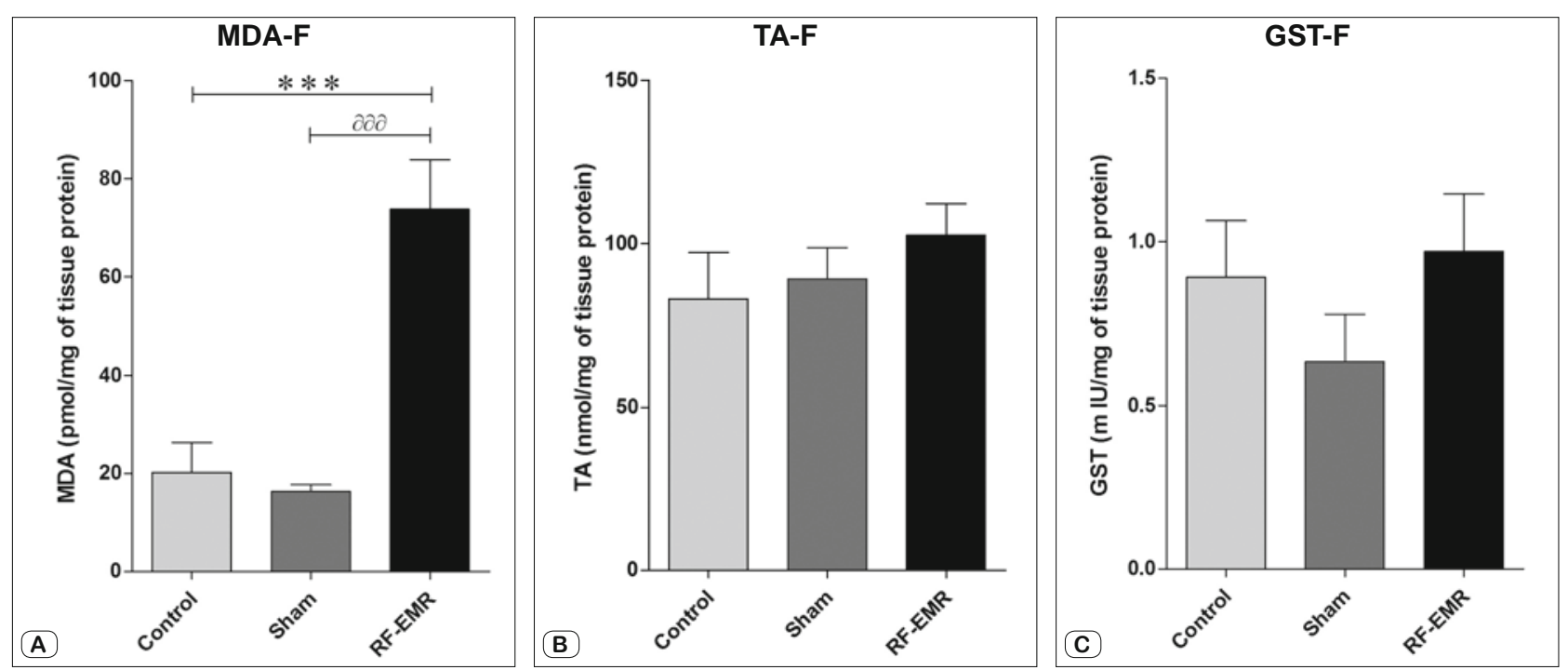

Fig. 3. Levels of thiobarbituric acid reactive substances (A), total antioxidants (B), and Glutathione S-transferase activity (C), in the frontal cortex. TBARS level were increased $\sim 4$ times in RF-EMR exposed groups when compared to control/sham groups. TA and GST activity did not show such significant difference when compared to controls. (Note: MDA-F - Malondialdehyde levels in frontal cortex, TA-F - Total antioxidants in frontal cortex, and GST-F - Glutathione S-transferase activity in frontal cortex respectively). *** $\mathbf{p}<0.001, \partial \partial \partial \mathrm{p}<0.001$.
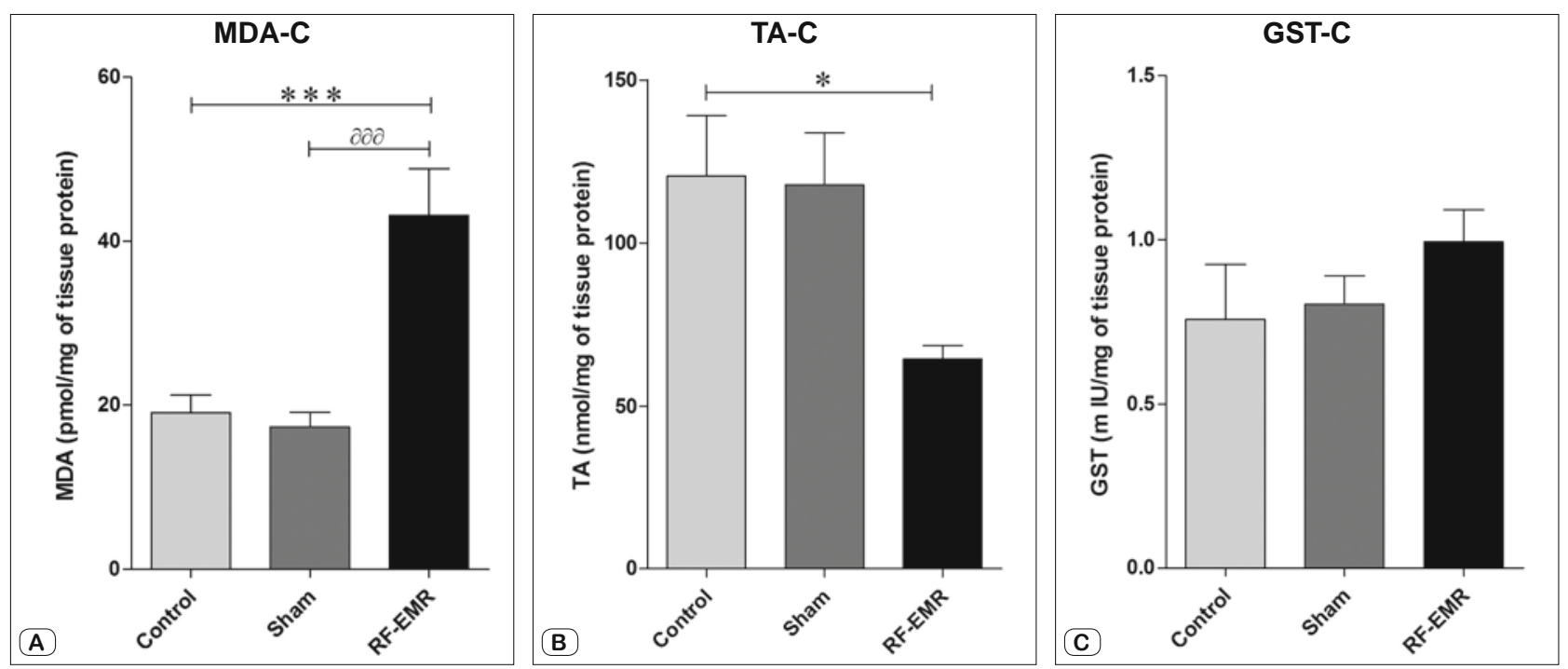

Fig. 4. Levels of thiobarbituric acid reactive substances (A), total antioxidants (B), and Glutathione S-transferase activity (C), in the cerebellum. Elevated levels of TBARS were seen in cerebellar tissue of RF-EMR-exposed rats. TA levels were decreased but GST activity in cerebellum of rats exposed to RF-EMR did not show any significant difference from controls. (Note: MDA-C - Malondialdehyde levels in cerebellum, TA-C - Total antioxidants in cerebellum, and GST-C - Glutathione S-transferase activity in cerebellum respectively). ${ }^{* *} \mathbf{p}<0.001, \partial \partial \partial \mathrm{p}<$ $0.001, * \mathrm{p}<0.05$.

\section{Biochemical indexes in the hippocampus}

The hippocampus of RF-EMR exposed rats showed elevated levels (two times) of TBARS when compared to control/sham groups (Fig. 2A: Control/Sham vs. RF-EMR; ** $\mathrm{p}<0.01, \partial \partial \mathrm{p}<$ 0.01; ANOVA, Tukey's post hoc tests). TA in all groups was not significantly different from each other (Fig. 2B). GST activity was also significantly decreased in RF-EMR-exposed rats when compared to control/sham groups (Fig. 2C: Control vs. RF-EMR; ** $\mathrm{p}<0.01$, Sham vs RF-EMR; $\partial \mathrm{p}<0.05$, ANOVA, Tukey's post hoc tests).

\section{Biochemical indexes in the frontal cortex}

TBARS levels were maximally elevated in this region in comparison to other brain regions studied in the present study. Its level increased $\sim 4$ times in RF-EMR exposed rats when compared to control/sham rats (Fig. 3A: Control/Sham vs. RF-EMR; *** $\mathrm{p}<0.001, \partial \partial \partial \mathrm{p}<0.001$; ANOVA, Tukey's post hoc tests). TA in RF-EMR group did not show such significant difference when compared to control/sham rats (Fig. 3B). GST activity also remained unaltered in this brain region (Fig. 3C). 

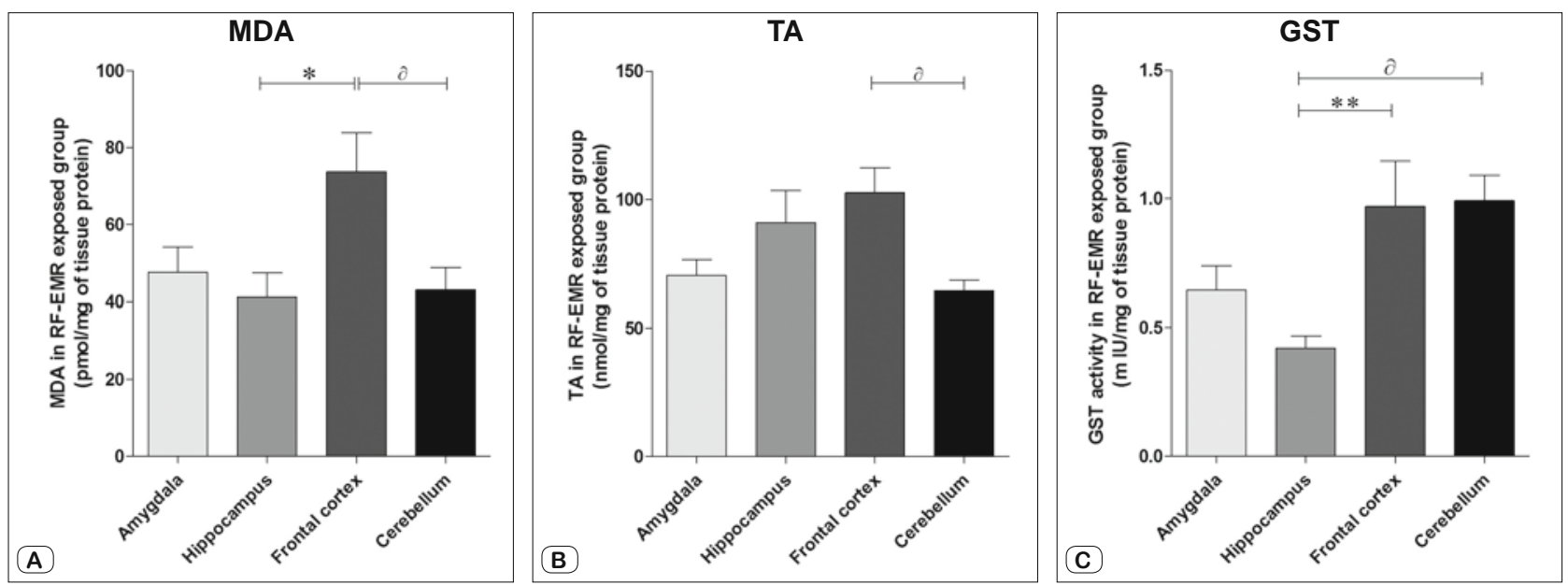

Fig. 5. Comparison of thiobarbituric acid reactive substances levels (A), total antioxidants (B), and Glutathione S-transferase activity (C) in different brain regions studied. The MDA levels were maximally elevated in the frontal cortex when compared to all other brain regions studied. TA was found to be significantly reduced in the cerebellum and GST activity was significantly reduced in the hippocampus. The magnitude of oxidative stress varies in different brain regions studied. ${ }^{*} \mathrm{p}<0.05, \partial \mathrm{p}<0.05, * * \mathrm{p}<0.01$.

\section{Biochemical indexes in the cerebellum}

Elevated levels of TBARS were seen in cerebellar tissue of RF-EMR exposed rats. In control group, the TBARS levels were $\sim 20 \mathrm{pmol} / \mathrm{mg}$ of tissue protein, but in RF-EMR group, the level was increased to $\sim 50 \mathrm{pmol} / \mathrm{mg}$ of tissue protein (Fig. 4A: Control/Sham vs. RF-EMR; *** $\mathrm{p}<0.001, \partial \partial \partial \mathrm{p}<0.001$; ANOVA, Tukey's post hoc tests). TA was also decreased in the cerebellum of RF-EMR exposed rats, but this difference was found to be statistically significant only with control group (Fig. 4B: Control vs. RFEMR; ${ }^{*} \mathrm{p}<0.05$; ANOVA, Tukey's post hoc tests). GST activity in cerebellum of RF-EMR-exposed rats was slightly elevated compared to control/sham groups but it was not significant (Fig. 4C).

\section{$V$. Comparison of biochemical indexes in different brain regions of RF-EMR-exposed rats}

Statistical analysis of various biochemical parameters in different brain regions of RF-EMR-exposed rats revealed that MDA levels were maximally elevated in the frontal cortex when compared to all other regions studied (Fig. 5A: Hippocampus vs. Frontal cortex; ${ }^{*} \mathrm{p}<0.05$, Frontal cortex vs. Cerebellum; $\partial \mathrm{p}<0.05$; ANOVA, Tukey's post hoc tests). TA was significantly reduced in cerebellum when compared to other regions studied (Fig. 5B: Frontal cortex vs. Cerebellum; $\partial \mathrm{p}<0.05$, ANOVA, Tukey's post hoc tests). TA was also reduced in amygdala compared to hippocampus and frontal cortex but this difference was not statistically significant (Fig. 5B). GST activity was significantly decreased in the hippocampus of RF-EMR-exposed group compared to all other regions studied (Fig. 5C: Hippocampus vs. Frontal cortex; ** $\mathrm{p}<$ 0.01; Hippocampus vs. Cerebellum; $\partial \mathrm{p}<0.05$; ANOVA, Tukey's post hoc tests,). Its activity was also reduced in the amygdala but this reduction was not statistically significant compared to other brain regions studied (Fig. 5C). RF-EMR exposure induced oxidative stress in rat brain by increasing lipid peroxidation and by decreasing antioxidant defense mechanisms. The magnitude of oxidative stress varies in different brain regions studied. In other words, it affects different brain regions differently.

\section{Discussion}

Reactive oxygen species (ROS) is a collective term that describes chemical species that are formed upon incomplete reduction of oxygen. These includes the superoxide anion $\left(\mathrm{O}_{2}^{-}\right)$, hydrogen peroxide $\left(\mathrm{H}_{2} \mathrm{O}_{2}\right)$ and hydroxyl radical (HO'). ROS are generated as byproducts of normal cellular metabolism. However, mammalian cells are equipped with both enzymatic and non-enzymatic antioxidant defense to minimize the cellular damage caused by interaction between cellular constituents and ROS (24).

Antioxidants are responsible for removing free radicals, scavenging ROS or their precursors, inhibiting formation of ROS and binding metal ions needed for catalysis of ROS generation (25). Several conditions are known to disturb the balance between ROS production and cellular defense mechanisms. This imbalance can result in cell dysfunction and destruction resulting in tissue injury. The increase in level of ROS in the cell could be due to their increased production or decreased destruction by antioxidants.

Upon critically evaluating the results obtained in the current study, it is evident that all brain regions studied had an elevated TBARS concentration in RF-EMR exposed rats compared to control and sham rats. An elevated level of TBARS is an index of lipid peroxidation in these regions. It was maximally elevated in the frontal cortex (Fig. 5A) and in amygdala when compared to other brain regions of RF-EMR exposed rats. This indirectly points to the fact that $900 \mathrm{MHz}$ RF-EMR exposure induced either generation of excess ROS or decreased removal of ROS in different brain regions.

Determining TA may help to identify conditions affecting the oxidative status in vivo (e.g. exposure to reactive oxygen species and antioxidant supplementation). In other words, its measurement will help to assess the redox status in an organism. Our result 
shows that TA was decreased in the amygdala and cerebellum of RF-EMR-exposed groups in comparison to control and sham. This is an important finding in view of the simultaneous deleterious effects of free radicals on the brain. It is well known that decreased antioxidants result in increased lipid peroxidation. One explanation for the observed lower level of TA in RF-EMR-exposed rats may lie in increased utilization of antioxidants in the process of scavenging the free radicals. In contradiction to this, in other brain regions studied, the TA values remained unaltered.

The glutathione transferase (GST; also known as glutathione Stransferase) is a major phase II detoxification enzyme found mainly in the cytosol. In addition to its role in catalyzing the conjugation of electrophilic substrates to glutathione (GSH), it has peroxidase and isomerase activities. It can inhibit the Jun $\mathrm{N}$-terminal kinase (thus protecting cells against $\mathrm{H}_{2} \mathrm{O}_{2}$-induced cell death), and it is able to bind non-catalytically to a wide range of endogenous and exogenous ligands (26). When we analyze the activity of GST in various brain regions, it is evident that its activity in RF-EMRexposed group was found to be different in various regions. In the present experiment, GST activity was decreased in the hippocampus and amygdala of RF-EMR-exposed rats in comparison to the control groups. It may be suggested that due to RF-EMR exposure, there is some reduction in antioxidant defense capacity (especially those involving glutathione) in these brain regions. Thus the RFEMR exposure makes brain more susceptible to other toxic effects. In brief, it is suggested that the RF-EMR exposure induced an imbalance in ROS production and cellular defense in various brain regions. How to explain these changes remains to be solved.

Neurons are largely depending on oxidative phosphorylation for energy and this makes them more vulnerable to oxidative stress compared to other cells. The metabolic activity of the brain is extremely high and the demand for oxygen consumption is also high with approximately $2 \%$ of the oxygen being converted into superoxide anion radicals $\left(\mathrm{O}^{2 \cdot-}\right)$ and hydrogen peroxide (27). In the current study, the rats were continually exposed (whole body exposure) to radiation for an hour per day, and this was continued for 28 days. This chronic exposure could have altered the oxidantantioxidant balances in the brain and lead to oxidative stress.

The current study is different from others in the fact that we report the magnitude of oxidative stress due to RF-EMR exposure in different brain regions. Also, it was evident from the current results that oxidant-antioxidant balance was altered significantly in prefrontal cortex and amygdala, while these brain regions regulate the emotionality (28) of organism. The biochemical changes in these regions could be read along with altered emotionality (18) seen in rats after RF-EMR exposure. Recent reports demonstrate that RF-EMR affects emotional learning and memory (8) regulated by basolateral amygdala and medial prefrontal cortex (29). RF-EMR also affected the hippocampal dependent spatial memory functions in rats $(30,31)$ and in mice (7). From the current results, it is evident that the biochemical indexes in these brain regions (amygdala, hippocampus) were also altered. In the light of these facts we believe that one of the reasons for behavioral alteration seen in rats after RF-EMR exposure is due to oxidative stress induced by RF-EMR in these brain regions. Further studies are necessary to elucidate what could be the other underlying cause (other than oxidative stress) for behavioral and other effects seen in RF-EMR-exposed animals.

The effect of RF-EMR-induced oxidative stress is a concern due to reasons as follows: 1 ) It could interfere with the learning and memory processes (32); 2) It may also accelerate various neurodegenerative diseases (33); 3) It might impair and oxidize DNA, lipids, sugars and proteins and consequently result in dysfunction of these molecules within cells and finally bring on cell death in various organs (34); 4) It could be a probable tumor promoter $(35,36)$.

The biological effects of mobile phone radio-frequency electromagnetic radiations (RF-EMR) are reported by many. But controversies remain in many of these reports. Oktem et al have reported that $900 \mathrm{MHz}$ radiation induced renal impairments demonstrated by an increase in tissue MDA, urine N-Acetyl-Beta-D-Glucosaminidase (NAG) levels, decreased superoxide dismutase (SOD), catalase (CAT), and glutathione peroxidase (GSH-Px) activities but melatonin treatment ameliorated oxidative tissue injury in rat kidney (16). A study undertaken to investigate the influence of electromagnetic radiation $(900 \mathrm{MHz})$ on oxidant and antioxidant levels in rabbits showed that serum SOD activity was increased, and serum nitric oxide (NO) levels were decreased in EMR-exposed animals compared to the sham group. No change was found in the levels of NO, MDA, and activities of adenosine deaminase, xanthine oxidase, catalase, myeloperoxidase, glutathione peroxidase in the serum and brains of either group (17). In another study, guinea pigs were exposed to $890-915 \mathrm{MHz}$ radiation (217-Hz pulse rate, 2-W maximum peak power, SAR $0.95 \mathrm{w} / \mathrm{kg}$ ) from a cellular phone for $12 \mathrm{hr} /$ day (11-h 45-min stand-by and 15-min spiking mode) for 30 days found that the MDAlevels were increased, glutathione (GSH) level and CAT enzyme activity were decreased, and vitamins A, E and $\mathrm{D}$ (3) levels did not change in the brain tissues of RF-EMR-exposed guinea pigs. Furthermore, the levels of MDA, vitamins A, D(3) and $\mathrm{E}$, as well as CAT enzyme activity increased, whereas GSH level decreased in the blood of RF-EMR-exposed animals (37).

Mobile phone safety recommendations depend only on 'sinusoidal wave' emitted from the cell phone when it is idle. At idle mode or stand-by mode, the RF-EMR exposure is negligible and hence it might not be harmful at this level. But chronic exposure to the 'carrier signal' emitted when phone is in 'ON' mode would have induced the above mentioned biological effects in rats.

Several attempts have been made to explain the possible mechanisms of action of electromagnetic radiation on the brain. Mobile phone radiation could have several effects on brain functions. These include thermal effects, non-thermal or specific effects, or cumulative effects (both thermal and non-thermal). The specific effects could be the culprit in inducing the above mentioned effects. Currently we are not sure about that as there is no concrete experimental evidence of mobile phone-specific effects. Further studies are warranted to prove which effect is inducing a change in various biochemical parameters in different brain regions of rats.

\section{Conclusion}

Results of the current study clearly demonstrate that, RF-EMR exposure for a period of one month induced excessive lipid per- 
oxidation and decreased antioxidants defense status in different brain regions of rats. We also conclude that oxidative stress could be one of the underlying mechanisms for behavioral alterations seen in rats after RF-EMR exposure. It is advisable to have a precautionary measure to reduce continuous and chronic exposure to RF-EMR from mobile phone and other RF-EMR emitting devices.

\section{References}

1. World Health Organization; "Electromagnetic fields and public health: mobile phones", Fact sheet №193, June 2011. http://www.who.int/mediacentre/factsheets/fs193/en/.

2. International Agency for Research on Cancer of World Health Organization; "IARC classifies radiofrequency electromagnetic fields as possibly carcinogenic to humans", press release № 208, 31 May 2011. http://www.iarc. fr/en/media-centre/pr/2011/pdfs/pr208_E.pdf.

3. Irmak MK, Oztas E, Yagmurca M, Fadillioglu E, Bakir B. Effects of electromagnetic radiation from a cellular telephone on epidermal Merkel cells. J Cutan Pathol 2003; 30 (2): 135-138.

4. Al-Damegh MA. Rat testicular impairment induced by electromagnetic radiation from a conventional cellular telephone and the protective effects of the antioxidants vitamins C and E. Clinics (Sao Paulo) 2012; 67 (7): 785-792.

5. Eșmekaya MA, Seyhan N, Ömeroğlu S. Pulse modulated $900 \mathrm{MHz}$ radiation induces hypothyroidism and apoptosis in thyroid cells: a light, electron microscopy and immunohistochemical study. Int J Radiat Biol 2010; 86 (12): 1106-1116.

6. Ntzouni MP, Stamatakis A, Stylianopoulou F, Margaritis LH. Shortterm memory in mice is affected by mobile phone radiation. Pathophysiology 2011; 18 (3): 193-199.

7. Fragopoulou AF, Miltiadous P, Stamatakis A, Stylianopoulou F, Koussoulakos SL, Margaritis LH. Whole body exposure with GSM 900MHz affects spatial memory in mice. Pathophysiology 2010; 17 (3): 179-187.

8. Narayanan SN, Kumar RS, Potu BK, Nayak S, Bhat PG, Mailankot M. Effect of radio-frequency electromagnetic radiations (RF-EMR) on passive avoidance behaviour and hippocampal morphology in Wistar rats. Ups J Med Sci 2010; 115 (2): 91-96.

9. Maskey D, Pradhan J, Aryal B, Lee CM, Choi IY, Park KS, Kim SB, Kim HG, Kim MJ. Chronic 835-MHz radiofrequency exposure to mice hippocampus alters the distribution of calbindin and GFAP immunoreactivity. Brain Res 2010; 1346: 237-246.

10. Thomas S, Benke G, Dimitriadis C, Inyang I, Sim MR, Wolfe R, Croft RJ, Abramson MJ. Use of mobile phones and changes in cognitive function in adolescents. Occup Environ Med 2010; 67 (12): 861-866.

11. Kwon MS, Vorobyev V, Kännälä S, Laine M, Rinne JO, Toivonen T, Johansson J, Teräs M, Lindholm H, Alanko T, Hämäläinen H. GSM mobile phone radiation suppresses brain glucose metabolism. J Cereb Blood Flow Metab 2011; (12): 2293-2301.

12. Cui K, Luo X, Xu K, Ven Murthy MR. Role of oxidative stress in neurodegeneration: recent developments in assay methods for oxidative stress and nutraceutical antioxidants. Prog Neuropsychopharmacol Biol Psychiat 2004; 28: 771-799.

13. Halliwell B. Role of free radicals in the neurodegenerative diseases: therapeutic implications for antioxidant treatment. Drugs Aging 2001; 18: 685-716.

14. Jing J, Yuhua Z, Xiao-qian Y, Rongping J, Dong-mei G, Xi C. The influence of microwave radiation from cellular phone on fetal rat brain. Electromagn Biol Med 2012; 31 (1): 57-66.

15. Dasdag S, Akdag MZ, Ulukaya E, Uzunlar AK, Ocak AR. Effect of mobile phone exposure on apoptotic glial cells and status of oxidative stress in rat brain. Electromagn Biol Med 2009; 28 (4): 342-354.

16. Oktem F, Ozguner F, Mollaoglu H, Koyu A, Uz E. Oxidative damage in the kidney induced by $900-\mathrm{MHz}-\mathrm{emitted}$ mobile phone: protection by melatonin. Arch Med Res 2005; 36 (4): 350-355.
17. Irmak MK, Fadillioglu E, Güleç M, Erdogan H, Yagmurca M, Akyol O. Effects of electromagnetic radiation from a cellular telephone on the oxidant and antioxidant levels in rabbits. Cell Biochem Funct 2002; 20 (4): 279-283.

18. Narayanan SN, Kumar RS, Paval J, Kedage V, Bhat MS, Nayak S, Bhat PG. Analysis of emotionality and locomotion in radio-frequency electromagnetic radiation exposed rats. Neurol Sci 2012 Sep 14. (Epub ahead of print)

19. Glowinski J, Iverson LL. Regional studies of catecholamines in the rat brain. I. The disposition of (3H)norepinephrine, $(3 \mathrm{H})$ dopamine and $(3 \mathrm{H})$ DOPA in various regions of the brain. J. Neurochem 1966; 13: 655.

20. Bradford MM. A rapid and sensitive method for the quantification of microgram quantities of proteins utilizing the principle of protein dye binding. Anal Biochem 1976; 72: 248-254.

21. Satoh K. Serum lipid peroxide in cerebrovascular disorders determined by a new colorimetric method. Clin Chim Acta 1978; 90: 37-43.

22. Koracevic G, Djordjevic V, Andrejevic S, Cosic V. Method for the measurement of antioxidant activity in human fluids. J Clin Pathol 2001; 54 (5): 356-361.

23. Beutler E. Red cell metabolism. In: A manual of biochemical method. Grune, Stratton, editors. New York: 1984, p. 8-78.

24. Powers SK, Jackson MJ. Exercise-induced oxidative stress: cellular mechanisms and impact on muscle force production. Physiol Rev 2008; 88 (4): $1243-1276$

25. Gilgun-Sherki, Y, Melamed E, Offen, D. Oxidative stress induced-neurodegenerative diseases: the need for antioxidants that penetrate the blood brain barrier. Neuropharmacol 2001; 40: 959-975.

26. Sheehan D, Meade G, Foley VM, Dowd CA. Structure, function and evolution of glutathione transferases: implications for classification of non-mammalian members of an ancient enzyme superfamily. Biochem J 2001; 360 (Pt 1): 1-16.

27. de Moura MB, dos Santos LS, Van Houten B. Mitochondrial dysfunction in neurodegenerative diseases and cancer. Environ Mol Mutagen 2010; 51 (5):3 91-405.

28. Canteras NS, Resstel LB, Bertoglio LJ, Carobrez Ade P, Guimarães FS. Neuroanatomy of anxiety. Curr Top Behav Neurosci 2010; 2: 77-96.

29. Laviolette SR, Grace AA. Cannabinoids Potentiate Emotional Learning Plasticity in Neurons of the Medial Prefrontal Cortex through Basolateral Amygdala Inputs. J Neurosci 2006; 26 (24): 6458-668.

30. Hao D, Yang L, Chen S, Tong J, Tian Y, Su B, Wu S, Zeng Y. Effects of long-term electromagnetic field exposure on spatial learning and memory in rats. Neurol Sci 2012 Feb 24. (Epub ahead of print)

31. Narayanan SN, Kumar RS, Potu BK, Nayak S, Mailankot M. Spatial memory performance of Wistar rats exposed to mobile phone. Clinics (Sao Paulo) 2009; 64 (3): 231-234.

32. Alzoubi KH, Khabour OF, Salah HA, Abu Rashid BE. The Combined Effect of Sleep Deprivation and Western Diet on Spatial Learning and Memory: Role of BDNF and Oxidative Stress. J Mol Neurosci 2012 Sep 7. (Epub ahead of print)

33. Fridovich I. Fundamental aspects of reactive oxygen species, or what's the matter with oxygen? Ann N Y Acad Sci 1999; 893: 13-18.

34. Mohsenzadegan M, Mirshafiey A. The immunopathogenic role of reactive oxygen species in Alzheimer disease. Iran J Allergy Asthma Immunol 2012; 11 (3): 203-216.

35. Aravalli RN, Cressman EN, Steer CJ. Cellular and molecular mechanisms of hepatocellular carcinoma: an update. Arch Toxicol 2012 Sep 25. (Epub ahead of print)

36. Kamendulis LM, Jiang J, Xu Y, Klaunig JE. Induction of oxidative stress and oxidative damage in rat glial cells by acrylonitrile. Carcinogenesis 1999; 20 (8): 1555-1560.

37. Meral I, Mert H, Mert N, Deger Y, Yoruk I, Yetkin A, Keskin S. Effects of 900-MHz electromagnetic field emitted from cellular phone on brain oxidative stress and some vitamin levels of guinea pigs. Brain Res 2007; 1169: 120-124.

Received December 24, 2012. Accepted February 28, 2014. 